\title{
Pessoa, filósofo
}

Nuno Filipe Ribeiro

Universidade Nova de Lisboa

\section{I - O espólio filosófico de Pessoa e os «milhares de filosofias»}

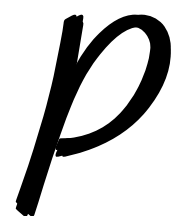

essoa não foi apenas poeta, escritor de ficções e crítico literário. Fernando Pessoa pretendeu também ser filósofo. No espólio deste autor encontramos uma multiplicidade de fragmentos destinados a futuras obras filosóficas que este pensador projectou mas que deixou inacabadas. Entre os diversos escritos que foram deixados da arca de Pessoa é possível identificar uma pluralidade de projectos e textos que se constituem como testemunhos do seu interesse pela tradição filosófica - os «milhares de filosofias», como este autor lhes chamou. É precisamente isso que lemos no seguinte trecho de um fragmento deixado por Fernando Pessoa:

Milhares de teorias, grotescas, extraordinárias, profundas, sobre o mundo, sobre o homem, sobre todos os problemas que pertencem à metafísica atravessaram o meu espírito. Tive em mim milhares de filosofias das quais - como se fossem reais - nem mesmo duas concordariam. ${ }^{2}$

2 LOPES, 1993, p. 402: "Thousands of theories, grotesque, extraordinary, profound, on the world, on man, on all problems that pertain to metaphysics have passed through my mind. I have had in me thousands of philosophies not any two of which - as if they were real - agreed." 
Esses «milhares de filosofias» foram mantidos em segredo por Pessoa e ficaram inéditos no decurso da vida do autor. Não é sem razão que na Tabacaria de Álvaro de Campos lemos: «Tenho feito filosofias em segredo que nenhum Kant escreveu.» ${ }^{3}$ No entanto, as indicações presentes no espólio de Pessoa permitem-nos revelar esta faceta da produção pessoana.

O espólio de Pessoa, catalogado na Biblioteca Nacional de Lisboa [BNP] sob a designação de «E3» [Espólio 3], encontra-se dividido em envelopes e compreende mais de vinte e sete mil documentos. Cada envelope está classificado com um número, uma designação e contém uma quantidade variável de documentos. Entre os diversos envelopes do espólio Pessoa encontramos quatorze envelopes filosóficos com 1428 documentos. Existem cinco envelopes $\left(15^{1}, 15^{2}, 15^{3}, 15^{4}\right.$ e $\left.15^{5}\right)$ com a designação «Filosofia», um (15A) classificado como «Filosofia-Metafísica», quatro $\left(15 \mathrm{~B}^{1}, 15 \mathrm{~B}^{2}, 15 \mathrm{~B}^{3}\right.$ e $\left.15 \mathrm{~B}^{4}\right)$ designados como «FilosofiaPsicologia» e, finalmente, quatro $(22,23,24$ e 25$)$ intitulados «Textos Filosóficos». No entanto, se é um facto que a divisão do espólio filosófico em envelopes nos permite identificar núcleos de textos filosóficos, este facto levanta um problema de índole topográfica. Por um lado, nem todos os textos filosóficos se encontram nos envelopes de índole filosófica e, por outro lado, os envelopes filosóficos contêm documentos que não são filosóficos. Assim, num envelope com a designação "Páginas de Estética e de Teoria e Crítica Literárias" [envelope 19] encontramos, por exemplo, um texto sobre a filosofia de Nietzsche intitulado «Friedrich Nietzsche» [BNP/ E3 - 19-99] ${ }^{4}$ e num envelope com a designação "Textos Filosóficos" [envelope 23] existe um texto com o título «De Profundis» [BNP/E3-23-66], publicado na selecção de textos filosóficos,

${ }^{3}$ CAMPOS, 2002, p. 322.

${ }^{4}$ PESSOA, 1994a, p. 333-334. 
intitulada Textos Filosóficos de Fernando Pessoa, realizada por António de Pina Coelho, ${ }^{5}$ mas que, no entanto, é uma tradução de um excerto do De Profundis de Óscar Wilde.

Os envelopes com a designação "Filosofia-Psicologia» [envelopes $15 \mathrm{~B}^{1}$ a $15 \mathrm{~B}^{4}$ ] são também um caso especial no espólio de Pessoa. Embora estes envelopes estejam classificados sob a designação de «filosofia», contêm escritos que, em grande parte, Pessoa classifica sob a designação de «Microsofia: a Ciência do Diminuto» [«Microsophy: the Science of the Minute»] [BNP/E3, 24-120], ${ }^{6}$ que é um termo criado pelo autor para designar áreas do conhecimento, tais como a frenologia, a fisionomia, assim como outras pequenas ciências relacionadas com a psiquiatria e não um grupo de textos que deva ser considerado sob a designação de "Filosofia". Assim, tendo todos estes aspectos em consideração, os textos filosóficos de Pessoa podem ser classificados em quatro grupos.

O primeiro grupo de textos filosóficos compreende os livros filosóficos inacabados. No espólio de Pessoa existem diversos documentos com projectos feitos pelo próprio Pessoa destinados a livros concebidos por este autor. Esses projectos encontram-se divididos em diversos tópicos, correspondentes a vários capítulos ou linhas de pensamento que Pessoa pretendia desenvolver em livros por si projectados. ${ }^{7}$ Muitos desses

${ }^{5}$ PESSOA, 1994b, p. 227-228.

${ }^{6}$ PESSOA, 2006, p. 167.

${ }^{7}$ Em muitos casos, Pessoa constrói mais do que um projecto para um mesmo núcleo de fragmentos, de tal modo que um conjunto de textos pode ser reagrupado de acordo com esquemas diversos. Isto é absolutamente evidente no caso dos contos deste autor, os quais passam não só por títulos diferentes, mas, em muitos casos começam por ser escritos numa língua (principalmente em inglês) e, no decurso da sua evolução, continuam a ser escritos noutra. Nos textos filosóficos existem também casos semelhantes. 
tópicos correspondem a diversas páginas que existem no espólio de Pessoa. Com efeito, a designação dos tópicos presente em muitos dos projectos corresponde ao título e às indicações de uma multiplicidade de páginas espalhadas ao longo do espólio de Pessoa. Entre os documentos com projectos de Pessoa, encontramos os projectos de livros filosóficos. O seguinte texto redigido por Pessoa em inglês e os seus diversos tópicos, que correspondem a um projecto destinado a um livro filosófico, constituem um exemplo de um projecto filosófico de Pessoa:

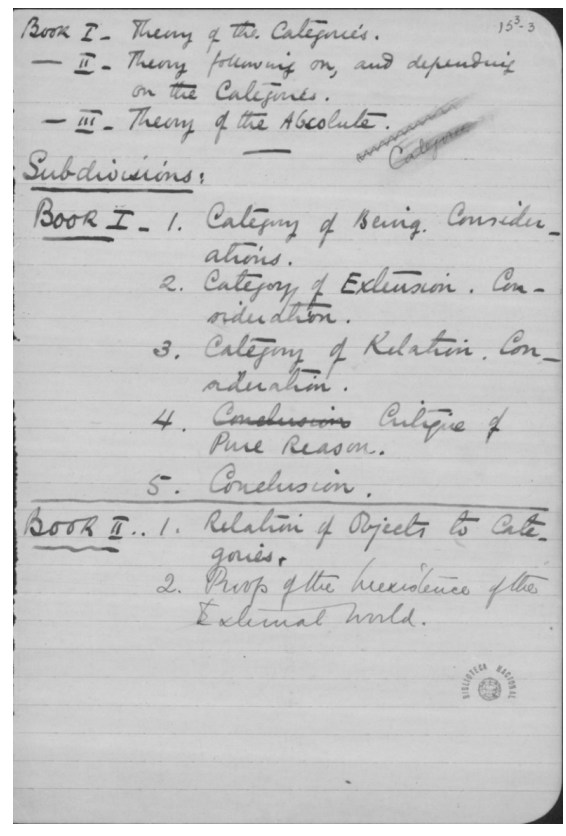

Book I - Theory of the Categories. [Book] II - Theory following on, and depending on the Categories.

[Book] III-Theory of the Absolute.

\section{Subdivisions:}

BookI-1. Category of Being. Considerations. 2. Category of Extension. Considerations.

3. Category of Relation. Consideration.

4. $<$ Conclusion $>$ Critique of Pure Reason.

5. Conclusion.

\section{Book II .. 1. Relation of Objects to} Categories.

2. Proof of the Inexistence of the External World.

$\left[\mathrm{BNP} / \mathrm{E} 3,15^{3}-3^{\mathrm{r}}\right]$

O segundo grupo de escritos filosóficos corresponde aos ensaios e pequenas produções. Existem vários géneros de ensaios com extensões diversas e que discutem o mais variado tipo de assuntos, autores e movimentos filosóficos. Por 
exemplo, no envelope $15^{4}$ existe uma série de dois documentos que correspondem a um ensaio inglês inacabado intitulado Essay on the Idea of Cause [BNP /E3, 15 4 -99 a 100: Anexo A]. Este ensaio constitui apenas um exemplo da pluralidade de ensaios e pequenas produções presentes ao longo do espólio de Pessoa.

O terceiro grupo de textos corresponde às notas de leitura de Pessoa. Pessoa tinha o hábito de tomar notas das suas leituras, muitas das quais se encontram conservadas no espólio deste autor. Isto acontecia a maior parte das vezes porque Pessoa lia livros que não eram seus, mas cuja referência queria manter. Estas notas são de crucial importância, não só para traçar as várias influências filosóficas na obra de Pessoa, mas também porque muitas dessas notas estão na origem de alguns dos seus escritos filosóficos. Assim, podemos distinguir dois tipos de notas: primeiro, as notas acríticas, que compreendem todas aquelas notas que têm meras indicações ou citações de livros filosóficos que Pessoa leu; segundo, as notas críticas, que, para além das referências e citações de livros, englobam também algumas considerações de Pessoa sobre aquilo que leu. No envelope 22 existe um exemplo de uma nota de leitura crítica, como se pode verificar no seguinte texto escrito por Pessoa em inglês sobre a definição aristotélica de metafísica:

Notes 1.

Aristotle defines metaphysics: (1) science of first principles and of first causes and (2) science of being as being [qua being]?

Also defined "the science of the absolute."

Metaphysics contains 3 great theories: 1) Theory of knowledge, 2) theory of being, 3) theory of the first principle of knowledge and of being.

Critique. It contains great theories indeed. These are: 1) Theory of knowledge, 2)?

[BNP/E3, 22 -73: fac-simile a seguir] 


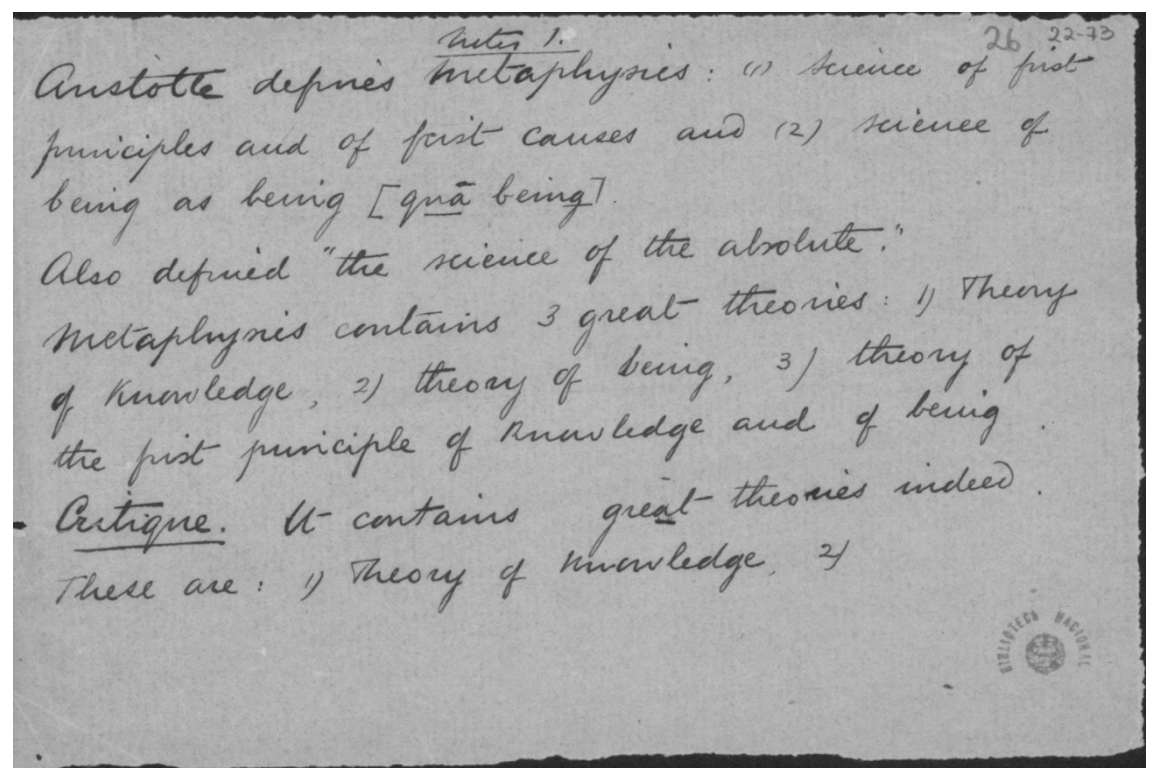

Finalmente, o quarto grupo de textos filosóficos corresponde às páginas filosóficas autónomas. Este tipo de textos engloba todos aqueles escritos que lidam com a filosofia ou com assuntos filosóficos, mas que não correspondem a nenhum dos outros grupos filosóficos, nem têm qualquer relação material directa com os outros escritos filosóficos. O espólio de Fernando Pessoa contém inúmeras páginas deste tipo.

Para além destes quatro grupos, pode ainda ser identificado um outro grupo de documentos filosóficos. Esse grupo de documentos corresponde ao projecto de criação de diálogos filosóficos. No envelope 24 existe um documento precisamente intitulado «Diálogos filosóficos» [BNP/E3, 24-96a $]^{8}$ e que contém um trecho de discussão sobre os argumentos acerca da existência de Deus. No entanto, os documentos relativos aos diálogos

8 PESSOA, 1994b, p. 129-130. 
filosóficos, presentes no espólio de Pessoa, são bastantes escassos e, por outro lado, o conteúdo e o estilo do texto intitulado «Diálogos filosóficos» encontram-se bastante próximos das ficções e contos deste autor. No espólio de Pessoa encontramos, por exemplo, um documento com a indicação

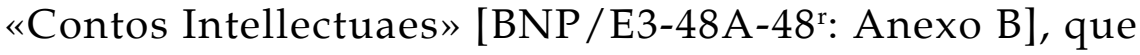
poderá corresponder a um desenvolvimento dos diálogos filosóficos. Isto leva-nos a concluir que, muito provavelmente, os diálogos filosóficos não terão passado da fase de projecto e que Pessoa terá reaproveitado o conteúdo destinado a esses diálogos para construir os seus contos e ficções, os quais, muitas vezes, são mais dialogados do que narrados.

\section{II - A produção filosófica de Pessoa e a multiplicidade de personalidades}

A criação de uma pluralidade de personalidades na obra de Fernando Pessoa tem inúmeras consequências no que respeita à circunscrição do âmbito da produção filosófica deste autor. No decurso da sua produção literária, Pessoa cria uma multiplicidade de personalidades entre as quais os heterónimos são as mais conhecidas. Um heterónimo é uma personalidade com uma biografia, um nome e uma obra inteiramente autónomos das demais personalidades. A cada heterónimo corresponde a fabricação de um estilo próprio. A fabricação de uma multiplicidade de heterónimos, corresponde à produção de uma multiplicidade de estilos.

Contudo, para além dos heterónimos, Pessoa cria ainda um conjunto de semi-heterónimos e de outras personalidades subalternas que poderão ser classificadas como sub-heterónimos. A diferença entre um heterónimo e um semi-heterónimo consiste no facto de que enquanto o heterónimo tem um estilo literário inteiramente autónomo, o semi-heterónimo escreve no 
mesmo estilo natural do autor real da escrita. É isso que Pessoa nos diz num fragmento destinado a um texto intitulado Ficções do Interlúdio a respeito do semi-heterónimo Bernardo Soares: "[...] Bernardo Soares, distinguindo-se de mim por suas ideias, seus sentimentos, seus modos de ver e de compreender, não se distingue de mim pelo estilo de expor $[\ldots]^{\prime \prime 9}$

Os sub-heterónimos desempenham, por outro lado, um papel inteiramente diferente dos heterónimos e dos semiheterónimos. Estas personalidades têm por função dominante a tradução e divulgação das obras dos heterónimos e de outros autores portugueses. Thomas Crosse e I. I. Crosse são dois exemplos deste tipo de personalidades.

No entanto, a produção de personalidades na obra de Fernando Pessoa não se esgota na criação dos heterónimos, semi-heterónimos e sub-heterónimos. A primeira aparição pública dos heterónimos de Pessoa ocorre em 1915 com a publicação do Opiário e da Ode Triunfal no primeiro número da revista literária Orpheu. Contudo, existe todo um trabalho de criação pré-heteronímica que antecede o surgimento dos heterónimos. Com efeito, Fernando Pessoa escreve sob o nome de mais de setenta personalidades literárias, ${ }^{10}$ a maior parte das quais produz as suas obras ou fragmentos de obras no período pré-heteronímico.

No que diz respeito aos pré-heterónimos, a produção de textos filosóficos encontra-se maioritariamente centralizada nos escritos de Charles Robert Anon e de Alexander Search. No espólio de Fernando Pessoa existe, por exemplo, um caderno com a indicação «Nº I.1. Charles R. Anon.» [BNP/E3, 13A-1 e

9 PESSOA, 2007, p.153.

${ }^{10}$ Em Pessoa por Conhecer, numa secção intitulada "1.1 - Dramatis Personae", Teresa Rita Lopes conta setenta e duas personalidades de Fernando Pessoa. Cf. LOPES, 1990, p. 167-169. 
ss.], que contém diversas páginas filosóficas atribuíveis a essa personalidade. Entre essas páginas consta, por exemplo, o projecto de um livro intitulado "“The World as Power."» [BNP/ E3, $13 \mathrm{~A}-1^{\mathrm{r}}$ : Anexo C], correspondente a inúmeros fragmentos dispersos ao longo do espólio de Pessoa. Relativamente a Alexander Search encontramos em The Transformation Book a referência a um projecto intitulado «The Philosophy of Rationalism» [BNP/E3 - 48C - 2r: Anexo D] que poderá ser interpretado como um dos títulos pensados, numa determinada fase da produção filosófica de Pessoa, para o conjunto de fragmentos intitulados Rationalism.

No que diz respeito às personalidades heteronímicas, a produção filosófica tem como principal preocupação a questão da definição do conceito de metafísica. De Álvaro de Campos existe um texto precisamente intitulado «O que é a metafísica?», publicado em 1924 no número 2 da revista Atena, que constitui uma contra-resposta ao ensaio "Atena», publicado pelo ortónimo no primeiro número dessa revista. António Mora participa igualmente nessa discussão, como se pode verificar pelo conjunto de fragmentos destinados a um ensaio intitulado "Introdução ao Estudo da Metafísica». ${ }^{11}$

Contudo, a questão da circunscrição dos textos filosóficos de Pessoa no âmbito da criação de uma multiplicidade de personalidades tem uma dificuldade acrescida. Se é verdade que Pessoa cria uma multiplicidade de textos assinados sob o nome das mais diversas personalidades literárias, também é um facto que no espólio de Pessoa encontramos vários textos sem qualquer atribuição autoral explícita ou implícita. Assim, os textos do espólio deste autor podem ser distinguidos em duas classes: os textos assinados ou incluídos em projectos

${ }^{11}$ Cf. MORA, 2002, p. 321-331. 
destinados a determinadas personalidades de Pessoa; segundo, os textos que não se encontram assinados. O segundo tipo de textos constitui aquilo que se pode designar de textos anónimos de Pessoa e compreende muito provavelmente a maior parte dos textos redigidos por Pessoa. É no âmbito dos textos anónimos de Pessoa que encontramos a maior variedade de projectos e fragmentos de livros, de pequenas produções e de ensaios filosóficos de Fernando Pessoa, discutindo os mais variados autores, desde Platão a Bergson, passando por considerações relativas a movimentos como o materialismo, o positivismo e o idealismo, bem como aos conceitos filosóficos de ser e de livre arbítrio.

\section{III - Pessoa - «poeta animado pela filosofia» e Pessoa-filósofo}

Os reflexos do interesse de Pessoa pela filosofia encontramse espelhados ao longo de toda a criação literária deste autor. Com efeito, a tematização da importância da filosofia e o emprego de conceitos filosóficos adquire expressão nas variadas produções pessoanas, desde a poesia e os contos até alguns dos textos teóricos de cariz político ou estético. Por exemplo, em O Guardador de Rebanhos de Alberto Caeiro encontramos a problematização, crítica e discussão de algumas noções filosóficas como os conceitos de metafísica, de alma e de Deus. ${ }^{12}$ Outro exemplo do eco de conceitos filosóficos em textos teóricos de cariz extra-filosófico encontra-se presente num texto de índole política intitulado A Aliança Ibérica. Nesse texto lemos:

${ }^{12}$ Veja-se em especial o poema V de O Guardador de Rebanhos. CAEIRO, 2001, p. 29-32. 
Criar uma nova literatura, uma nova filosofia - esse é o primeiro passo. Foi dado em Portugal, em filosofia sobretudo, por Leonardo Coimbra, um dos três grandes filósofos da Europa contemporânea (os outros dois são Bergson e Eucken). ${ }^{13}$

Estes e outros exemplos presentes na obra de Fernando Pessoa são suficientes para dar a conhecer um Pessoa-«poeta animado pela filosofia» ["poet animated by philosophy»], ${ }^{14}$ que este autor afirmou ter sido e efectivamente chegou a ser, mas não para pôr em evidência um Pessoa-filósofo. Com efeito, a presença de conceitos filosóficos ao longo das diversas produções poéticas, ficcionais e de teoria política ou estética de Fernando Pessoa constituem apenas o indício das leituras filosóficas que terão ocupado este autor em diferentes períodos da sua vida. Mas a relação de Pessoa com o pensamento filosófico não se circunscreve às menções a autores, movimentos ou conceitos filosóficos presentes nos seus mais variados textos poéticos, ficcionais ou de teoria política e estética. Para além de poeta «animado pela filosofia», Pessoa pretendeu ser também um autor filosófico. Os diversos escritos filosóficos presentes no espólio de Pessoa são o testemunho da actividade filosófica deste autor. Assim, para além do Pessoa-«poeta animado pela filosofia» é possível descortinar entre os escritos do espólio deste autor um Pessoa-filósofo. O cultivo do estilo e dos diversos géneros filosóficos foi, para Fernando Pessoa, um dos momentos do desenvolvimento de uma multiplicidade de estilos. A escrita filosófica constitui para Pessoa uma forma de literatura. Para além dos fragmentos de livros e ensaios filosóficos, encontramos entre as pequenas produções filosóficas de Pessoa inúmeros projectos de artigos e tratados. Os projectos de livros,

13 PESSOA, 1986, p. 994.

14 PESSOA, 2003, p. 18. 
tratados, ensaios e de artigos do espólio de Pessoa correspondem à multiplicidade de géneros filosóficos que este autor pretendia e que, na verdade, chegou a desenvolver, ainda que de um modo fragmentário. Deste modo, o estilo filosófico e os diversos géneros nele incluídos foram para Pessoa a ocasião para o desenvolvimento de uma forma de literatura, isto é, de uma literatura que deixou inacabada, mas cujos projectos e indicações presentes ao longo de todo o espólio nos permitem reconstruir.

\section{Adenda}

\section{Publicação e edições dos textos filosóficos de Pessoa (1968-2010)}

A publicação dos textos filosóficos de Pessoa conta com três marcos principais. O primeiro corresponde à edição em dois volumes intitulada Textos Filosóficos de Fernando Pessoa, publicada em 1968 por António de Pina Coelho na Editorial Ática. ${ }^{15}$ Esta edição, que foi objecto de sucessivas reedições, constitui-se como uma primeira apresentação dos escritos filosóficos do espólio de Pessoa. No entanto, apresenta várias lacunas. Em primeiro lugar, contém apenas uma pequena selecção de textos filosóficos. A selecção e transcrição dos textos filosóficos, presentes nessa edição, encontram-se circunscritas a quatro envelopes filosóficos (envelopes: 22, 23, 24 e 25), o que

${ }^{15}$ A referência da edição é: Fernando Pessoa, Textos Filosóficos de Fernando Pessoa (estabelecidos e prefaciados por António Pina Coelho), Lisboa, Ática, 1968. A última reedição desta obra foi feita em 2006: Fernando Pessoa, Textos Filosóficos de Fernando Pessoa (estabelecidos e prefaciados por António Pina Coelho), Lisboa, Editorial Nova Ática, 2006. No essencial, a obra não sofre nenhuma alteração, nem de organização, nem de conteúdo. 
representa somente uma pequena percentagem dos documentos filosóficos de Pessoa, englobando sobretudo os mais fáceis de transcrever. Em segundo lugar, esta edição não dispõe de um critério crítico para a selecção, transcrição e organização dos textos. No prefácio de Pina Coelho à edição dos Textos Filosóficos de Fernando Pessoa lemos:

Para a arrumação dos textos aduzidos, seguimos, com os riscos inerentes, o critério adoptado por Georg Rudolf Lind e Jacinto do Prado Coelho nos dois últimos volumes de prosa de Fernando Pessoa, por eles coligidos e publicados pela Ática - distribuindo-os por assuntos. ${ }^{16}$

Porém, esta distribuição dos textos por assuntos, correspondentes a núcleos temáticos, não obedece nem a um princípio temático de organização estabelecido por Pessoa, nem a um projecto do espólio concebido por este autor para reunir um grupo de textos filosóficos, tratando-se, por conseguinte, de um critério eminentemente subjectivo e que não atende à especificidade dos textos no contexto dos projectos filosóficos de Fernando Pessoa.

O segundo marco relativo aos textos filosóficos de Pessoa corresponde ao livro Fernando Pessoa. Moral, Regras de Vida, Condições de Iniciação publicado por Pedro Teixeira Mota em 1988. Neste livro existe a compilação de diversos textos relativos à filosofia moral de Pessoa entre os quais, um conjunto de escritos destinado ao Essay on Free-Will, um dos principais projectos filosóficos de Pessoa. No entanto, esta compilação de Teixeira da Mota, para além de compreender apenas um número reduzido de textos de filosofia moral de Fernando Pessoa, inclui textos que escapam ao âmbito das reflexões filosóficas deste autor, abrangendo, de igual modo,

${ }^{16}$ PESSOA, 1994b, p. XXI. 
alguns escritos relativos ao domínio do pensamento esotérico de Pessoa.

Para além das duas referências mencionadas, encontramos no livro Pessoa Inédito, ${ }^{17}$ organizado por Teresa Rita Lopes e publicado em 1993, um capítulo com 22 páginas, contendo uma selecção de textos filosóficos feita por José Gil. No prefácio introdutório à selecção de textos, José Gil faz apelo à necessidade de uma edição crítica que estabeleça critérios objectivos e rigorosos de selecção e organização dos textos filosóficos. ${ }^{18} \mathrm{No}$ entanto, a selecção feita por José Gil tem somente a intenção de iniciar o público à presença de reflexão filosófica no espólio pessoano, ou, utilizando as palavras do próprio autor:

[...] os textos apresentados destinam-se apenas a dar um exemplo da riqueza do espólio inédito, e da sua importância para o estudo de tantos problemas por resolver do enigma pessoano. ${ }^{19}$

O período situado entre 1994 e 2010 é caracterizado por uma relativa escassez no que respeita à publicação dos textos filosóficos de Fernando Pessoa, que no essencial se resume a referências esparsas, fragmentárias e assistemáticas. Embora o número de dissertações sobre problemáticas filosóficas no pensamento deste autor tenha aumentado no decurso desse

17 LOPES, 1993. Ver especificamente as páginas 106-109 (2.13. "Onde o poeta se manifesta animado pela filosofia") e 397-420 («8. Um poeta animado pela Filosofia»).

${ }^{18}$ Com efeito, na introdução de José Gil, lemos o seguinte a propósito dos textos filosóficos: «Haverá a possibilidade de estabelecer períodos, quando uma edição crítica vier a separar o trigo do joio, a obra pessoal dos mimetismos, precisar influências.» AAVV, 1993, p. 107.

${ }_{19}$ AAVV, 1993, p. 109. 
período, o interesse pelo espólio filosófico e pela edição dos textos filosóficos não cresceu de um modo proporcional ao número de dissertações filosóficas a respeito do pensamento de Pessoa. Na sua maioria, as dissertações sobre Pessoa e a filosofia circunscrevem-se à produção poética, ficcional ou a textos de índole literária, esquecendo-se de que entre os projectos de Fernando Pessoa se encontra uma multiplicidade de escritos destinados a projectos filosóficos deste autor. 


\section{Anexos $^{20}$}

\section{Anexo A}

[BNP/E3, 154-99 a 100]

$\left[100^{\mathrm{r}}\right]^{21}$

1.22

A. S. ${ }^{23}$

${ }^{20}$ Os símbolos utilizados para a transcrição dos documentos que constituem os presentes anexos são:

[ ] intervenções ou acrescentos do editor

? espaço deixado em branco pelo autor

* leitura conjecturada

/ / lição dubitada pelo autor

$<>\quad$ segmento autógrafo riscado

$<>/$ substituição por superposição

$<>$ [? ] substituição por riscado e acrescento

[?] acrescento na entrelinha superior

${ }^{21}$ No espólio de Fernando Pessoa [BNP/E3] as referências $15^{4}-99^{r}$ e $99^{v}$ são catalogadas antes das referências $100^{\mathrm{r}}$ e $100^{\mathrm{v}}$. No entanto, parece tratar-se de um erro de catalogação pois as últimas são lógica e argumentativamente anteriores às primeiras.

$22<198>$, riscado no canto superior direito da página.

${ }^{23}$ As siglas «A. S.» poderiam sugerir que o Essay on the Idea of Cause se trataria de um texto de Alexander Search, cujas siglas seriam a abreviatura. Todavia, este ensaio é inspirado em e discute as teses do Essai sur le Livre Arbitre [CFP, 1-135] de Schopenhauer. As siglas «A.S.» poderão, por conseguinte, ser também interpretadas como a abreviatura de Arthur Schopenhauer. 


\section{Essay on the Idea of Cause. (extract).}

Schopenhauer has divided the idea of Cause into 3 other ideas properly speaking: Causation, excitation ${ }^{24}$ and motivation. The first occurs in the domain of the inorganic things; it is c[h]aracterized by its conformity to the first and to the second laws of Newton. The anterior modification corresponds always to the posterior modification; and action and reaction are equal. Excitation differs from causation in that there is no regular correspondence between the cause and the effect. Motivation is, according to Schopenhauer, causation acting through an intellect. Thus motivation has the character of finality. Every act ${ }^{25}$ has an end.

Let us examine closely these ideas and see how true they are and how far they go. In the first place it is quite true that causation in the inorganic, inanimate world is ${ }^{26}$ rigorously subject to law. Nay more, inanimate ${ }^{27}$ things are exempt, as far as we can see, from the law of differences, or, as Leibnitz calls it, the Law of indiscernibles. We are bound to admit that the same amount of heat applied at several times to the same bar of iron will produce in all cases a similar expansion. But?

We are quite justified in saying that if the same amount of heat could be trice ${ }^{28}$ applied [100v $]$ to the same bar of iron, it would at all times most ${ }^{29}$ certainly produce the same expansion.

\footnotetext{
${ }^{24}<$ motivation> excitation, no original.

$25<$ *hum $>$ [? act], no original.

${ }^{26}$ world $<,>$ is, no original.

${ }^{27}<$ the $>$ inanimate, no original.

${ }^{28}$ trice [? several times], no original. Escolhemos a primeira variante, uma vez que é aquela que mais coerente é com a sequência do texto. ${ }^{29}$ would [? at all times] most, no original.
} 
But, as far as we know, the same fact is not repeated exactly as it was, nor are there, we believe, two like things in the whole universe. The bar of iron we have used is not the bar of iron we use now, third other time. Its radio-activity is indeed infinitesimal; nevertheless it exists and an experiment cannot be the same. The bar of iron is the same and it is not the same. "Being is nothing", said Heraclitus, "and ${ }^{30}$ becoming is all."

(Certitude is mathematical and no more. There is no stability in physical things. I cannot divide a thing in half, I must make some mistake. No 2 things in the world being equal, it is clear that I cannot divide a thing correctly, that those two empirical halves cannot be equal. But mathematically all this is possible and true. It is true that 4 is the half of 8 and that one ${ }^{31}$ half 4 is always equal to the other half $4 .{ }^{32}$

[99']

In the first place then we have to make this objection: what Schopenhauer calls causation has nothing at all of cause. The transmission of heat to a bar of iron which results in the partition ${ }^{33}$ of the iron molecules, is no relation of cause to effect. It is nothing but a transmission of movement; nothing different from a billiard ball ${ }^{34}$ which hits another and stops, imparting to the other its movement. Again if at two diff[eren]t times I throw a stone with equal force ${ }^{35}$ - a pure hypothesis, of course -

$30<$ for $>$ [? and], no original.

${ }^{31}$ and [? that] one, no original.

${ }^{32}$ Embora com parêntesis no inicio, o texto não fecha o parágrafo com parêntesis, o que muito provavelmente se deverá a um lapso do autor.

${ }^{33}$ apartition, no original. Trata-se, muito possivlmente, de uma gralha do autor

${ }^{34}$ ball $<\mathrm{s}>$, no original.

35 force $<^{*} \mathrm{I}>$, no original. 
though in diff[eren]t directions, once against a tree, at another time along a clear field, ${ }^{36}$ it is obvious that even here there is no question of causation. However diverse ${ }^{37}$ the movement of the two stones, their movem[en]t, in regard to its quantity, is rigorously equal; it cannot but be so, being in both cases the product of an equal force. And a movement must perforce be the product and the continuation of another movement and this one the result of another and thus are linked all the forces and motions of the universe. The so called causation is nothing but the change from one movement to another, or from one form of movement to another form, or a change of media in the same movement.

[99v]

Example of football field. ${ }^{38}$

Consider now the form of Cause which Schop[enhauer] calls excitation. Here we have no longer a movement, for a movement would transmit itself equally. Yet it produces always

In me and in the player excitation ${ }^{39}$ has produced a different effect. It cannot then be a movement; it cannot be conceived as one. Let us consider excitation more closely than we have done. The nature of excitation is, it is said, that there is no real proportion ${ }^{40}$ between cause and effect (so to call them) or, better, between the anterior and the consequent modification.

\footnotetext{
${ }^{36}<\mathrm{f}>$ clear field, no original.

$37<$ diff $>$ diverse, no original.

${ }^{38}<$ Leiria $>$, palavra riscada escrita na parte superior central da página.

39 /man/ [? player] <movement $>$ excitation,l no original.

${ }^{40}$ no [? real] proportion, no original.
} 


\section{Anexo B}

[BNP/E3, 48A-48

Contos Intellectuaes.

e outros

1. A perda do hiate Nada.

2. O desapparecimento do professor Serzedas.

3. A morte do dr. Cerdeira.

4. Na casa de saúde de Cascaes.

5. Uma tarde clerical.

6. O circulo da vida.

7. Rosa-Cruz.

8. O Eremita da Serra Negra.

9. N'um bar de Londres.

10. Elogio histórico de D. Miguel Roupinho.

11. Notas de conversas com Carlo Malva.

12. A Tortura pela escuridão.

13. A experiencia do dr. Lacroix.

14. A porta.

15. Jacob Dermot.

16. Um jantar muito original. 


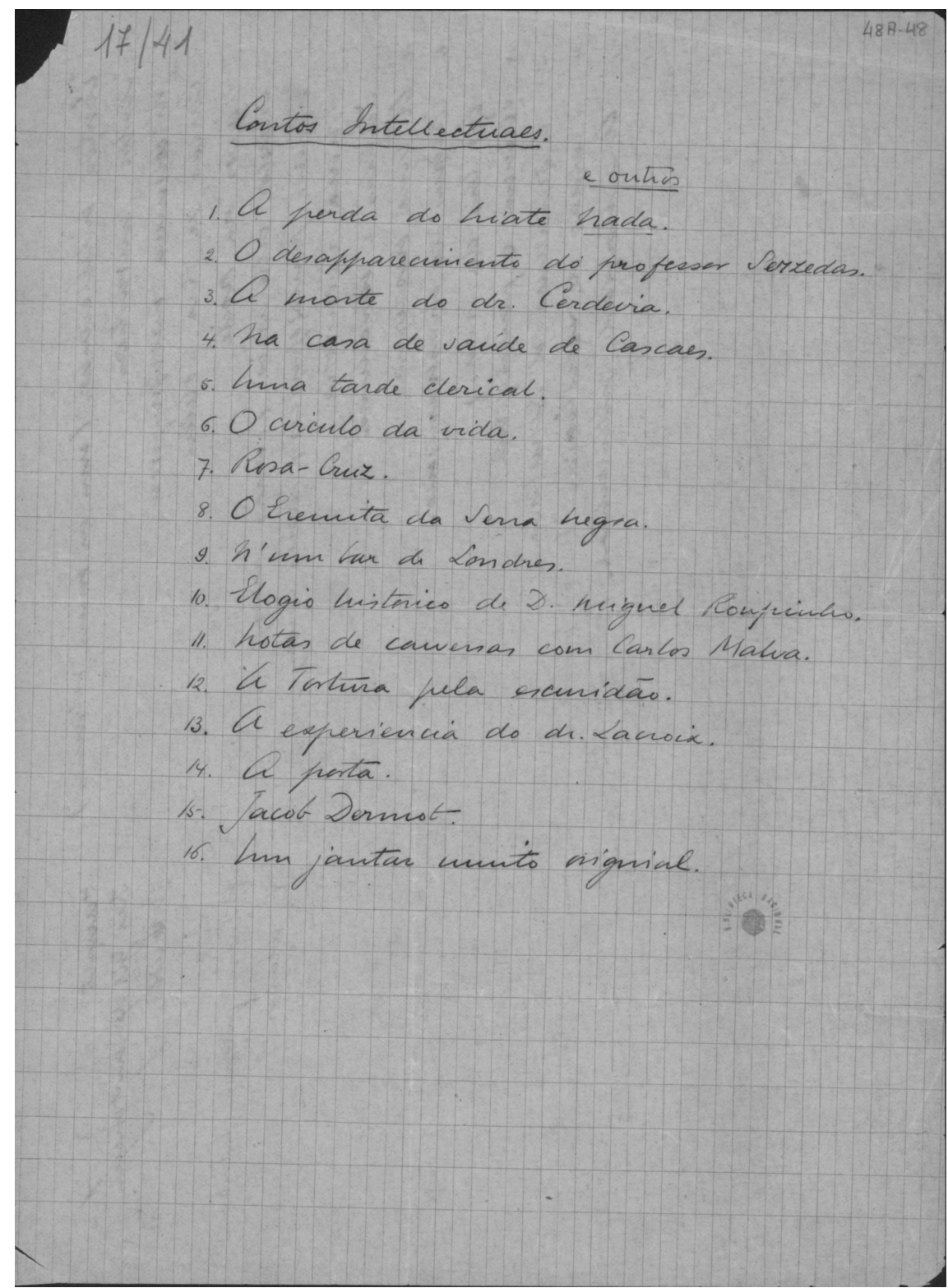

Fac-simile da lista de Contos Intellectuaes [BNP/E3, 48A-48 ] 


\section{Anexo C}

$\left[\mathrm{BNP} / \mathrm{E} 3,13^{\mathrm{a}}-1^{\mathrm{r}}\right]$

“The World as Power." ${ }^{41}$

I. Life is power.

Struggle for life.

I. The Individual.

II. The state.

\section{Heraclitus $^{42}$}

Being ${ }^{43}$ is nought; becoming is all. But then Being is Becoming. ${ }^{44}$ No for then Becoming is Being, ${ }^{45}$ and thereby nothing is solved. But if a thing really become(s) other ${ }^{46}$ than it is (appearance), it must have anteriorly the ${ }^{47}$ power to become. /Existence $/{ }^{48}$ is therefore not Becoming, Being (Existence) is Power.

The Process of Nature:

1. Power; 2. Movement; 3. Evolution;

4. Struggle for Life. 5. Selection.

${ }^{41}<$ The $>$ "The World as Power.", no original.

${ }^{42}$ Heraclitus <ôi åí>, no original.

${ }^{43}<\mathrm{b}>$ Being, no original.

${ }^{44}<\mathrm{b}>/ \mathrm{B} \backslash$ ecoming, no original.

${ }^{45}<\mathrm{b}>/ \mathrm{B} \backslash$ ecoming is $<\mathrm{b}>/ \mathrm{B} \backslash$ eing, no original.

${ }^{46}$ thing [? really] become(s) [? <at a>] other, no original.

${ }^{47}$ [? anteriorly] the, no original.

${ }^{48}<$ Being $>$ [? Existence ?], no original. 
Revista do CESP - v. 32, n. 48-jul.-dez. 2012

- those "The lived ar Power."

T. Life is hower.

Striggle for Life.

I. The howichal.

II. The state.

Herachios

Senig is nonght; becoming is all.

But then Senig is becoming. ho for theer becoming is Bering, ano therlby nothing is. solved. But if a tring heconved other thain it is Tappearance], it must have L the frover to become'. Buits is therefore not

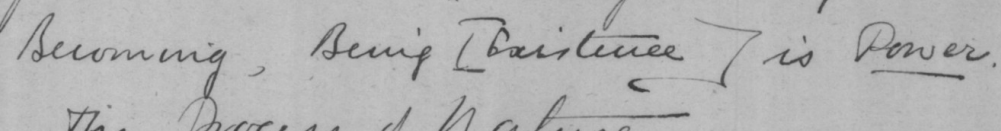

The proass of hature.

1. Pिver; 2. movenent; 3. Evolution,

4. Stingle for Life. Ś. Selechion.

Fac-simile do projecto do livro filosófico The World as Power [BNP $\left./ \mathrm{E} 3,13 \mathrm{~A}-1^{\mathrm{r}}\right]$

149 


\section{Anexo D}

$\left[\mathrm{BNP} / \mathrm{E} 3-48 \mathrm{C}-2^{\mathrm{r}}\right]$

Alexander Search

Born June 13th. 1888, at Lisbon.

Task: all not the province of the other three.

1. "The Portuguese Regicide and the Political Situation in Portugal."

2. "The Philosophy of Rationalism."

3. "The Mental Disorder(s) of Jesus."

4. "Delirium."

5. "Agony."

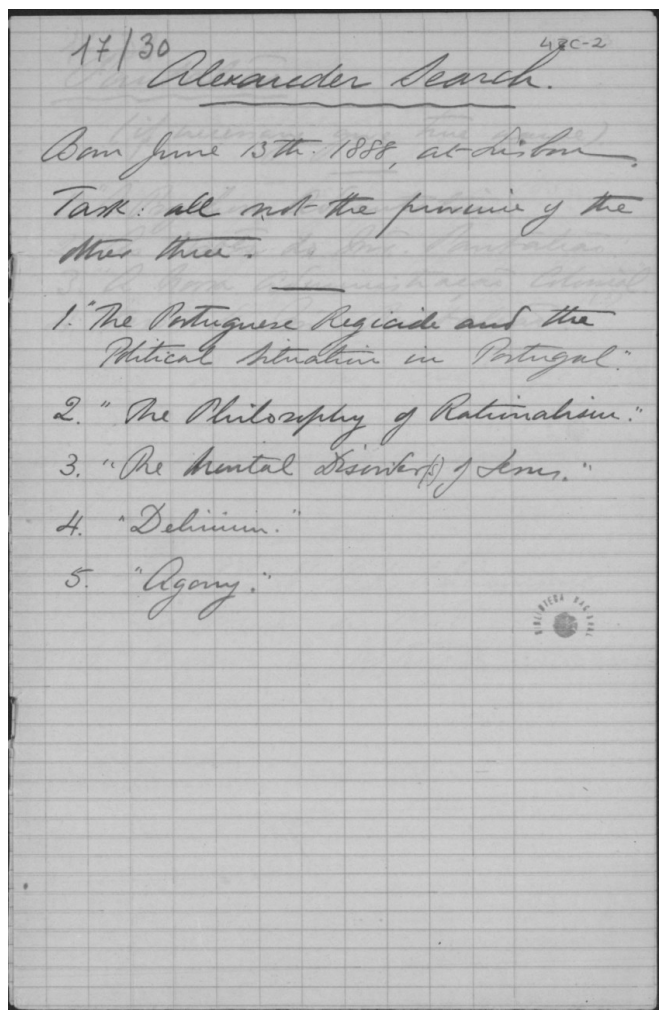




\section{Referências}

BOTTO, António. Canções. PIZARRO, Jerónimo; RIBEIRO, Nuno. (Ed.). Lisboa: Guimarães, 2010.

CAEIRO, Alberto. Poesia. MARTINS, Fernando Cabral; ZENITH, Richard. (Ed.). Lisboa: Assírio \& Alvim, 2001.

CAMPOS, Álvaro de. Poesia. LOPES, Teresa Rita. (Ed.). Lisboa: Assírio \& Alvim, 2002.

LOPES, Teresa Rita (Org.). Pessoa inédito. Lisboa: Livros Horizonte, 1993.

LOPES, Teresa Rita. Pessoa por conhecer. Vols. I e II. Lisboa: Editorial Estampa, 1990.

MORA, António. Obras de António Mora. Lisboa: Imprensa Nacional / Casa da Moeda, 2002.

MOTA, Pedro Teixeira da. Fernando Pessoa. Moral, regras de vida, condições de iniciação. Textos estabelecidos e comentados por Pedro Teixeira da Mota. Lisboa: Edições Manuel Lencastre, 1988.

PESSOA, Fernando. Crítica. MARTINS, Fernando Cabral. (Ed.). Lisboa: Assírio \& Alvim, 2000.

PESSOA, Fernando. Escritos autobiográficos, automáticos e de reflexão pessoal. ZENITH, Richard. (Ed.). Lisboa: Assírio \& Alvim, 2003.

PESSOA, Fernando. Escritos sobre génio e loucura. Lisboa: Imprensa Nacional Casa da Moeda, 2006.

PESSOA, Fernando. Ficções do interlúdio. MARTINS, Fernando Cabral. (Ed.). Lisboa: Assírio \& Alvim, 1998.

PESSOA, Fernando. Obra poética e em prosa. Vol. III. Introdução, organização, bibliografia e notas de António Quadros e Dalila Pereira da Costa. Porto: Lello \& Irmãos, 1986.

PESSOA, Fernando. Páginas de estética e de teoria e crítica literárias. Lisboa: Ática, 1994a. 
PESSOA, Fernando. Prosa intima e de autoconhecimento. Lisboa: Assírio \& Alvim, 2007.

PESSOA, Fernando. Textos filosóficos de Fernando Pessoa. Estabelecidos e prefaciados por António Pina Coelho. Vols I e II. Lisboa: Ática, 1994b.

\section{Resumo}

O presente artigo pretende evidenciar a dimensão filosófica da obra de Fernando Pessoa. No espólio de Pessoa encontramos uma multiplicidade de projectos e fragmentos destinados a obras filosóficas que este autor deixou inacabadas e que se constituem como o testemunho do seu interesse pelas mais variadas tradições filosóficas. Assim, através da análise do desenvolvimento dos diversos testemunhos filosóficos póstumos de Pessoa, pretendemos clarificar em que sentido é possível delinear o retrato de Pessoa enquanto filósofo.

\section{Abstract}

This article intends to clarify the philosophical dimension of Fernando Pessoa's work. In the Pessoa Archive one finds a multiplicity of philosophical projects and fragments, which Pessoa left unfinished and that testify his interest about the several philosophical traditions. Thus, through the analysis of the development of the various philosophical posthumous writings of Pessoa, we intend to outline the portrait of Pessoa as philosopher. 